\title{
Effect of cybotactic clusters on the elastic and flexoelectric properties of bent-core liquid crystals belonging to the same homologous series
}

\author{
R. Balachandran, ${ }^{1}$ V. P. Panov, ${ }^{1}$ J. K. Vij,${ }^{1,3, *}$ A. Lehmann, ${ }^{2}$ and C. Tschierske ${ }^{2}$ \\ ${ }^{1}$ Department of Electronic and Electrical Engineering, Trinity College, University of Dublin, Dublin 2, Ireland \\ ${ }^{2}$ Institute of Chemistry, Martin Luther University Halle-Wittenberg Kurt-Mothes Strasse 2, D-06120 Halle/Saale, Germany \\ ${ }^{3}$ School of Electronic and Electrical Engineering, Sungkyunkwan University, Suwon 440-476, Korea
}

(Received 5 June 2013; revised manuscript received 29 July 2013; published 17 September 2013)

\begin{abstract}
We report results of the splay $\left(K_{11}\right)$ and bend $\left(K_{33}\right)$ elastic constants and the effective flexoelectric coefficient of three bent-core liquid crystals belonging to a homologous series of 4-cyanoresorcinol bisbenzoates with varying chain lengths. Based on the results of x-ray scattering studies, one of the three compounds with a shorter chain length (C4) has few, if any, clusters present in its nematic phase and behaves quite normally, whereas the others two with longer chain lengths (C6 and C7) show the presence of cybotactic nematic phase with smectic $\mathrm{C}$ type clusters. These grow in size with a reduction in temperature. $K_{33}$ is found to be the least for $\mathrm{C} 7$, whereas it is weakly dependent on temperature. $K_{33}$ is somewhat higher for $\mathrm{C} 4$ and C6 and is almost independent of temperature. $K_{11}$ for C6 and C7 is higher by $20 \%$ to $50 \%$ than $\mathrm{C} 4$ depending on the temperature. $K_{11}$ increases linearly with a reduction in temperature for the three compounds. For C6 $K_{11}>K_{33}$ by a factor up to $\sim 2$ depending on the temperature, for $\mathrm{C} 4$ it is greater by a factor up to 1.3 , and for $\mathrm{C} 7$ it is greater by a factor of $\sim 2.5$. These results suggest that the clusters do not have any effect on $K_{11}$. The magnitude of the effective flexoelectric coefficient $e=\left(\left|e_{1}-e_{3}\right|\right)$ is measured by creating a uniform lying helix (ULH) configuration in a planar cell. By doping the bent-core system with a small wt \% of a chiral dopant, the ULH is obtained by cooling planar cells to the cholesteric phase under weak electric field. The effective flexoelectric coefficient is greater for the bent-core systems than for calamatics but it is much lower than would otherwise have been expected for such systems. $\left|e_{1}-e_{3}\right|$ for $\mathrm{C} 4>\mathrm{C} 6 \approx \mathrm{C} 7$ is greater by $20 \%$ to $25 \%$ than $\mathrm{C} 6$ and $\mathrm{C} 7$ at the same reduced temperature. These differences in the effective flexoelectric coefficient can easily arise from a difference in the chain lengths among the members of the series but if the presence of clusters were to have an influence on $\left|e_{1}-e_{3}\right|$, then these would reduce it, contrary to the expectations for the bent-core systems.
\end{abstract}

DOI: 10.1103/PhysRevE.88.032503

PACS number(s): 42.70.Df, 61.30.Gd

\section{INTRODUCTION}

The molecular structure of a liquid crystalline material is found to greatly influence its physical properties and its phase behavior. A well-known structure of a calamitic mesogen exhibiting nematic phase consists of a rigid core containing a number of phenyl rings (two or more) in the middle and usually one flexible terminal chain connected to one of its ends; the second end terminates in a polar group. In liquid crystals composed of banana shaped molecules, the rigid core is replaced by a bent aromatic core and is usually connected to flexible tails on both sides. Though bent-core molecules composed of intrinsically achiral molecules were first synthesized by Vorländer as early as the 1930s [1], their special properties were not realized until Niori et al. [2] and Link et al. [3] discovered polar switching and spontaneous breaking of the achiral symmetry in smectic phases. This demonstration led to intense research activity, carried out by several groups internationally [4]. This activity received a great boost in the last decade due to the outcome of exciting and new science that came out of these investigations. A variety of orthogonal smectic phases have recently been discovered in several bent-core systems [5] and several types of the nematic phase (differing in detail from each other) are also observed in some compounds synthesized by several groups [6]. Towards the end of the last and the beginning of the current decade,

\footnotetext{
*jvij@tcd.ie
}

the transition temperatures from the Isotropic-Nematic (I-N) phase have consistently been lowered, especially by the works of Tschierske et al. [7]. Though smectic phases of bent-core and calamitic systems offer huge potential for a range of applications, the commercially successful liquid crystal (LC) applications, so far, are mostly based on nematics. This is owing to a number of desirable properties of nematic phase such as its lower viscosity, greater response to the external electric and magnetic fields, and mechanical distortions, as well as to their fast recovery from defects and distortions [8]. One of the essential features of the nematic LCs is that the mechanical distortions of a group of molecules give rise to electric polarization (this is referred to as flexoelectricity). The phenomenon may yield much higher values for the flexoelectric coefficient for bent-core compounds that exhibit nematic phase than conventional calamitics [9]. These liquid crystals are called bent-core nematics (BCNs).

\section{FLEXOELECTRICITY}

The phenomenon of flexoelectricity or the flexoelectric effect was predicted by Meyer [10] some 40-odd years ago. This effect arises from a coupling between the elastic deformations and the electric polarization. Flexoelectric polarization can manifest itself even in nonpolar nematic LCs when subjected to splay or bend distortions of the director. In the direct flexoelectric effect, a gradient of the strain leads to macroscopic flexoelectric polarization $\mathbf{P}_{f}$ and in the converse flexoelectric effect, the external field $E$ leads to a 


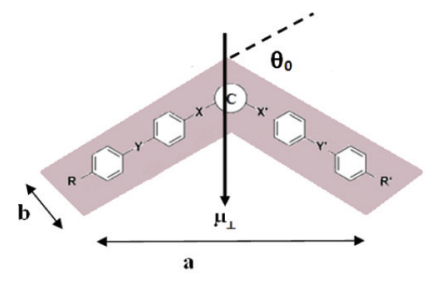

(a)

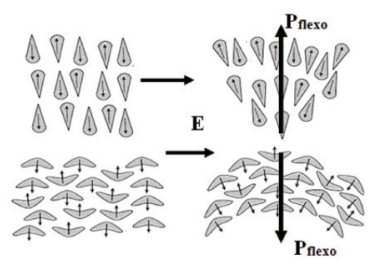

(b)
FIG. 1. (Color online) (a) A model of a bent-core molecule depicting the kink angle $\theta_{0}$ and the transverse dipole moment $\mu_{\perp}$, $\mathbf{a}$ and $\mathbf{b}$ denote the length and width of the molecule, respectively. (b) Illustration as to how electric field can induce flexoelectric polarization in drop-shaped (top right) and crescent/bend shaped (bottom right) molecules by splay and bend distortions of the liquid crystalline medium, respectively.

gradient in the stress curvature. Normally in the absence of external perturbations, the molecular arrangement is such that the net flexoelectric polarization is zero. For molecules with permanent dipole moments and asymmetric shapes, such as the pear/drop or crescent/banana shaped, this effect is caused by mechanical distortions or conversely by the application of electric field (see Fig. 1). The flexoelectric polarization [10] $\mathbf{P}_{f}$ is expressed in terms of the splay and bend flexoelectric coefficients and is given as follows:

$$
\mathbf{P}_{f}=e_{1} \mathbf{n}(\boldsymbol{\nabla} \cdot \mathbf{n})+e_{3} \mathbf{n} \times(\boldsymbol{\nabla} \times \mathbf{n}) .
$$

In Eq. (1), $e_{1}$ and $e_{3}$ are the flexoelectric coefficients corresponding to the splay and bend deformations, respectively. These are connected to the splay $\left(K_{11}\right)$ and bend $\left(K_{33}\right)$ elastic constants; $\mathbf{n}$ is the director field. For example, for the case when molecules fluctuate independently of each other, $e_{1}^{2}=\varepsilon_{\|}$ $K_{11}^{D}$ and $e_{3}^{2}=\varepsilon_{\perp} K_{33}^{D}$ [11]. $\varepsilon_{\|}$and $\varepsilon_{\perp}$ are the dielectric permittivities parallel and normal to $\pm \mathbf{n}$. The superscript $D$ on the elastic constants denotes constant electric displacement, $D$. In addition, $K_{11}$ is also connected to the threshold voltage for the Freederickz transition. These relations initially derived by Helfrich [12] are recently reviewed by Castles et al. [11]. The latter also put in maximum limits on the equalities between the flexoelectric coefficients and the elastic constants for the constant electric displacements.

Using the molecular statistical approach, Helfrich [12] derived an expression for the bend flexoelectric coefficient:

$$
e_{3}=\mu_{\perp} \frac{K_{33}}{2 k_{B} T} \theta_{o}\left(\frac{b}{a}\right)^{\frac{2}{3}} N^{\frac{1}{3}} .
$$

Here, $\mu_{\perp}$ is the molecular dipole moment perpendicular to the molecular long axis and is directed along the bow direction; $a$ and $b$ denote the length and the width of a molecule, respectively. $k_{B}$ is the Boltzmann constant and $T$ is the absolute temperature; $\theta_{0}$ is the kink angle. $N$ is the number density of molecules. Thus, in principle at least, we can design molecules that exhibit much greater bend flexoelectric coefficient in their nematic phase. For the bent-core systems, $\theta_{0}$ is $\sim 60^{\circ}$ and if the molecule is designed to have a reasonably large $\mu_{\perp}$, then, in principle at least, these systems can exhibit much higher bend flexoelectric coefficients provided $K_{33}$ is also not reduced. It is thus conceivable that due to its bent structure, the elastic constants and the flexoelectric coefficients acquire different values than calamitics. Equation (2) is also applicable to calamatics with the exception that the bent-core angle $\theta_{0}$ is of the order of $5^{\circ}$.

Harden et al. [9] determined $\left|e_{3}\right|$ for a banana system in its nematic phase using a method of measuring polarization caused directly by the mechanical distortions. They found $\left|e_{3}\right|$ to be higher by a factor of $10^{3}$ than calamitic LCs. However, the validity of this finding has been hotly debated $[11,13]$ as the result so far cannot be reproduced using other methods [11]. Irrespective of the correctness of this result, the finding in itself has led to intense experimental and theoretical investigations. If the results were eventually found to be correct, we would be able to design better flexoelectric devices for converting mechanical into electric energy using bent-core LCs. With this objective in mind, we set forth to determine independently the various elastic constants and the flexoelectric coefficients of the bent-core systems that exhibit nematic phase closer to the room temperature. We examine whether the observed cybotactic clusters present in the nematic phase of these systems affect flexoelectric properties since only one of the three bent-core LCs (C4) is found to be almost devoid of cybotactic clusters; the others two have cybotactic clusters which increase in size with a reduction in temperature.

However, a method for observing the direct flexoelectric effect is not easy to design nor is the experimental result easily interpretable since the mechanical distortions can make the ions migrate through the medium and could cause electroconvection arising from mechanical distortion. The mobility of ions can alter the measuring current since frequencies of 1 to $10 \mathrm{~Hz}$ for mechanical deformations used by Harden et al. [9] are too low and can easily result in mobility of ions contributing to the measured current. Only if the frequencies of the field were large enough would the ions not respond to the field. It will thus be difficult to separate out the two currents: caused by mobility of ions and by the flexoelectric polarization. Furthermore, the effect of sliding one substrate over the second as done by Harden et al. [9] can easily rupture the director field and can thus easily lead to erroneous results for the flexoelectric coefficient. Instead, most experiments revolve around using the converse flexoelectric effect $[12,14]$, where the measurement frequency, in principle, can be higher but, more importantly, it is known that the ions do not significantly affect the electro-optical response as the current is affected by the ions. The flexoelectric effect is related to the bulk flexoelectric torque, $\boldsymbol{\tau}$, arising from deformations of the director field brought about by the electric field and the flexoelectric response is connected to the measurement of this torque. This can be expressed as $\boldsymbol{\tau}=(\mathbf{n} \times \mathbf{h})$, where $\mathbf{h}$ is the bulk flexoelectric component of the molecular field and $\mathbf{n}$ is the director field [14-16]:

$$
\mathbf{h}=\left(e_{1}-e_{3}\right)[\mathbf{E}(\nabla \cdot \mathbf{n})-(\boldsymbol{\nabla} \otimes \mathbf{n}) \mathbf{E}]-\left(e_{1}+e_{3}\right)(\mathbf{n} \cdot \nabla) \mathbf{E} .
$$

From Eq. (3) it can be concluded that the bulk deformations can be obtained from a gradient in the distribution of either the nematic director caused by mechanical distortions or by the external electric field (uniform $E$ or gradient in $E$ or both) [16]. Hence, in the case when a uniform electric field is applied, the term involving the difference part $\left|\left(e_{1}-e_{3}\right)\right|$ contributes to the torque. The electric field gradient, if properly designed, can couple to the $\left|\left(e_{1}+e_{3}\right)\right|$ term in Eq. (3) [14-16] and hence 
$\left|\left(e_{1}+e_{3}\right)\right|$ can thus be found. Some of the commonly used techniques for finding $\left|\left(e_{1}-e_{3}\right)\right|$ include measurements of the in-plane tilt of the optic axis of a chiral nematic LC when the molecules are aligned in a uniform lying helical (ULH) configuration [17] or in a Twisted Nematic (TN)/Hybrid Aligned Nematic (HAN) cell, while the sum $\left|\left(e_{1}+e_{3}\right)\right|$ can be determined using HAN or $\pi$ cells [18]. The ULH configuration is easily realizable in achiral bent-core systems by doping with a commercially available chiral dopant with a significantly larger twisting power. This is rather a recent commercial chemical development. It is also found that ULH configuration is more easily obtainable with the bent-core nematics than with the conventional calamatics.

Bent-core compounds that exhibit nematic phase do have a range of other interesting properties. Some of these include intrinsic biaxiality [19] and the optical biaxiality measured directly [20] and induced biaxiality [21], a reversal in the sign of the elastic and dielectric anisotropies [22-25], and the observed chiral domains of the opposite polarity in C5 [26] and C9 [26]. For a widely studied conventional calamitic such as 4-cyano-4'-pentylbiphenyl (5CB), $K_{33}$ is greater than $K_{11}$ and the ratio of $K_{33} / K_{11}$ varies from 2 to 3 [27]. However, in some other calamitics the ratio is found to be $>1$ for small chain lengths, $\sim 1$ for intermediate chain lengths, and $<1$ for longer chain lengths. The ratio of $K_{33} / K_{11}$, in general, decreases as the chain length increases [28]. For a given chain length of the molecule, a decrease in the ratio $K_{33} / K_{11}$ is attributed to the presence of smecticlike clusters and the underlying smectic phases [28]. Schadt et al. designed new molecular systems to reduce $K_{33} / K_{11}$ of nonpolar alkenyl nematics by introducing double bonds in either or both of the end chains so as to make the nematic LCs switch faster by the external field [29]. However, in the bent-core systems that we studied, $K_{33}<K_{11}$ irrespective of the chain length or presence of the clusters. We can safely conclude that the bent shape of the molecules facilitates the bend distortion and is mainly responsible for determining it. Dodge et al. [30,31] showed that by mixing small amounts of bent-core systems in a calamitic nematic (8OCB) provided it is dissolvable, $K_{33}$ is significantly reduced. Kundu et al. [32] made similar findings for calamitic 8OCB mixed with different concentrations of bent-core LCs in the nematic phase but they additionally obtained somewhat anomalous results for the elastic constants. They found that closer to the nematic-smectic phase transition temperature both $K_{11}$ and $K_{33}$ first decrease and then increase slightly with a reduction in temperature, in contrast to both of these elastic constants increasing significantly closer to the nematicsmectic phase transition temperature in the host 8OCB. In recent investigations, measurement of these constants in pure BCNs in nematic phase has also yielded intriguing results, $K_{33}$ is found in most cases to be lower than $K_{11}$ [24,25,32-34].

Extraordinarily large values for the bend flexoelectric coefficient $\left|e_{3}\right|$ for $\mathrm{BCN}$ by a factor of $10^{3}$ greater than calamatics observed by Harden et al. [9] were suggested to arise from the smecticlike cluster formation in the nematic phase of these compounds [24,25]. Discrepancies in the results between the elastic and flexoelectric coefficients given in the literature prompted us to investigate properties of three BCN nematic LCs that belong to a homologous series. Such a comparison, in principle, should give a useful insight into the

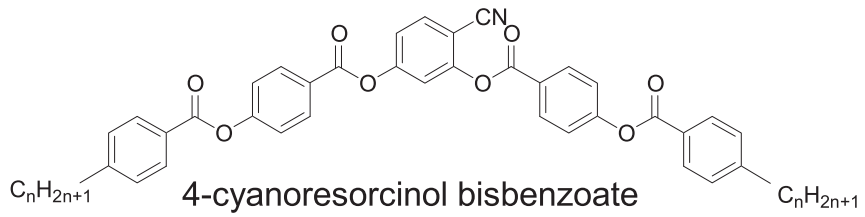

FIG. 2. Generic chemical structure of the bent-core compound 4-cyanoresorcinol bisbenzoates under investigation. In general length of the alkyl chain can be different on two sides, but here the alkyl chains are of the same length.

behavior of bent-core LCs in their nematic phase under the influence of external stimuli. We report studies on the splay and bend elastic constants, examine the ratio $K_{33} / K_{11}$ and the effective flexoelectric coefficient $\left|\left(e_{1}-e_{3}\right)\right|$ as a function of temperature in the nematic phase of the three compounds. All of the three BCNs have the same 4-cyanoresorcinol bisbenzoate core but these differ in chain lengths, $n=4$ (C4), $n=6$ (C6), and $n=7$ (C7) and thus these belong to a homologous series. The generic structure of the homologous series is shown in Fig. 2 and the phase transition temperatures are given in Table I. When cooled from the isotropic phase to lower temperatures, the materials exhibit I-N transition; $\mathrm{C} 4$ and $\mathrm{C} 7$ show a monotropic nematic phase while C6 exhibits an enantiotropic phase. X-ray scattering studies have shown that the smectic phase is not present in either of $\mathrm{C} 4$ or $\mathrm{C} 6$ even at low temperatures but smectic-C-like clusters are present in both $\mathrm{C} 6$ and $\mathrm{C} 7$ but these are much smaller or measurably absent in $\mathrm{C} 4$ [7]. The dielectric and electro-optic studies of $\mathrm{C} 4$ revealed that it exhibits $\Delta \varepsilon>0$ for lower frequencies and higher temperatures, whereas $\Delta \varepsilon<0$ for higher frequencies and lower temperatures [22]. The slow crystallization of C4 as observed is also being reported here. The rate of cooling is much lower compared to the DSC results presented in [7].

\section{EXPERIMENTAL METHODS}

\section{A. Dielectric anisotropy}

Sandwich LC cells were prepared using indium tin oxide (ITO) glass plates. The ITO coated glass substrates were spin coated with polymer aligning agents RN1175 (Nissan Chemicals, Japan) for planar and AL60702 (JSR, Korea) for homeotropic alignments. Cells were cured at appropriate temperatures to obtain planar and homeotropic aligned cells incorporating $\mathrm{C} 4, \mathrm{C} 6$, and C7. Cells for the homogeneous alignment were rubbed and assembled in such a way that the rubbing directions on the top and bottom substrates were antiparallel to each other. The cell thickness was determined using a spectrometer (Avaspec-2048) interfaced with a PC. The typical cell thickness used in the experiments varied from 5 to $10 \mu \mathrm{m}$. The empty capacitance of the cell was measured before filling it up with the material. Each cell was capillary filled with the sample by heating the empty cell to a temperature of the isotropic phase of the compound using a temperature controller (Eurotherm 2604). The parallel and perpendicular components of the dielectric permittivity, $\varepsilon_{\|}$and $\varepsilon_{\perp}$ were obtained from homeotropic and planar cells, respectively, using Novocontrol Broadband High Resolution dielectric spectrometer, Alpha-A Frequency Analyzer, interfaced with a PC. The reasons for 
TABLE I. Transition temperatures $\left(T /{ }^{\circ} \mathrm{C}\right)$ and the associated enthalpy values $(\Delta H / \mathrm{kJ} \mathrm{mol})$ given in square brackets of the 4-cyanoresorcinol bisbenzoates under study [7]. Cr, crystalline solid; Iso, isotropic liquid; $\mathrm{N}$, nematic phase similar to the ordinary nematic phases; NcybC, nematic phase composed of SmC type cybotactic clusters. Peak temperatures are taken from the first DSC heating curves $(10 \mathrm{~K} / \mathrm{min})$. The values in the round brackets refer to the monotropic transitions observed on cooling. In general, number of carbon atoms on the left ( $m$ ) of the core structure can be different than on the right $(n)$, but here $m=n$.

\begin{tabular}{lc}
\hline \hline$n$ & Phase transitions \\
\hline 4 & Cr $117[38.4]$ (N 107 [0.5]) Iso \\
6 & Cr 98 [37.1] NcybC 101 [0.5] Iso \\
7 & Cr 96 [35.5] (SmC 25 [0.3] CybC 41 [0.2]) NcybC 111 [0.7] Iso \\
\hline \hline
\end{tabular}

different relaxation dynamics for both $\varepsilon_{\|}$and $\varepsilon_{\perp}$ are discussed in detail by Jang et al. [23].

\section{B. Splay and bend elastic constants}

The optical retardation, $\delta$, of a planarly aligned sample was measured as a function of the electric field for different temperatures using a photoelastic modulator (PEM) setup [35] and a polarizing microscope. A helium-neon laser is a source of monochromatic light to the PEM setup and the signal from the detector is amplitude and phase locked using two lock-in amplifiers. The retardation data is obtained as a function of voltage for different temperatures by applying a sinusoidal voltage of $\sim 20 V_{p k}$ at a frequency of $1 \mathrm{kHz}$. The splay elastic constant, $K_{11}$, is determined from the threshold voltage $\left(V_{t h}\right)$ for the Freedericksz transition using the equation $K_{11}=\left(\frac{V_{t h}}{\pi}\right)^{2} \varepsilon_{o} \Delta \varepsilon$, where $\Delta \varepsilon$ is the dielectric anisotropy; $\Delta \varepsilon=\varepsilon_{\|}-\varepsilon_{\perp}$ and $\varepsilon_{0}$ is the permittivity of free space. The bend elastic constant, $K_{33}$, was obtained from a theoretical fit of the retardation data for a planar cell, for voltages greater than the threshold voltage, to Eqs. (4) and (5) [36,37] using an iterative procedure designed by us [35],

$$
\begin{gathered}
\frac{V}{V_{t h}}=\frac{2}{\pi} \sqrt{1+\gamma \sin ^{2}\left(\phi_{m}\right)} \int_{0}^{\frac{\pi}{2}} \sqrt{\frac{1+\kappa \sin ^{2}\left(\phi_{m}\right) \sin ^{2}(\psi)}{\left[1+\gamma \sin ^{2}\left(\phi_{m}\right) \sin ^{2}(\psi)\right]\left[1-\sin ^{2}\left(\phi_{m}\right) \sin ^{2}(\psi)\right]} d \psi,} \\
\delta=2 \pi \frac{n_{e} d}{\lambda}\left\{\frac{\int_{0}^{\frac{\pi}{2}} \sqrt{\frac{\left[1+\gamma \sin ^{2}\left(\phi_{m}\right) \sin ^{2}(\psi)\right]\left[1+\kappa \sin ^{2}\left(\phi_{m}\right) \sin ^{2}(\psi)\right]}{\left[1-\sin ^{2}\left(\phi_{m}\right) \sin ^{2}(\psi)\right]\left[1+\nu \sin ^{2}\left(\phi_{m}\right) \sin ^{2}(\psi)\right]}} d \psi}{\int_{0}^{\frac{\pi}{2}} \sqrt{\frac{\left[1+\gamma \sin ^{2}\left(\phi_{m}\right) \sin ^{2}(\psi)\right]\left[1+\kappa \sin ^{2}\left(\phi_{m}\right) \sin ^{2}(\psi)\right]}{\left[1-\sin ^{2}\left(\phi_{m}\right) \sin ^{2}(\psi)\right]}} d \psi}-\frac{n_{o}}{n_{e}}\right\},
\end{gathered}
$$

where $d$ is the cell thickness, $\Phi$ is the z-dependent tilt angle, $\Phi_{m}$ is the tilt angle in the middle of the cell and the other terms are defined as $\kappa=\left(K_{33} / K_{11}\right)-1, \gamma=\left(\varepsilon_{||} / \varepsilon_{\perp}\right)-1$, $v=\left(n_{e}^{2} / n_{o}^{2}\right)-1$, and $\sin (\Phi)=\sin \left(\Phi_{m}\right) \sin (\psi)$.

\section{Flexoelectric coefficients}

The measurement of the effective flexoelectric coefficient $\left|\left(e_{1}-e_{3}\right)\right|$ was carried out by exploiting the flexoelectro optic effect observed in the geometry of a ULH [38] obtained by doping the sample with a left-handed chiral dopant (S811 E. Merck, Korea) of large twisting power. This gives a negative sign of $\left(e_{1}-e_{3}\right)$ if we were to follow the normal convention given by Castles et al. [39] as opposed to the convention adopted by Salter et al. [40]. In general, ULH texture is largely unstable as it is incompatible with both homeotropic and planar alignments since the minimum free energy configuration of CLC corresponds to a Grandjean texture [41]. A method of obtaining ULH with the cholesteric helix parallel to the substrate involves cooling the sample from the isotropic phase to just below the I-cholesteric phase transition temperature under the application of a moderate field [38]. In some cases the device needs to be subjected to mechanical shearing in a direction perpendicular to the rubbing direction but it was not found necessary to do so as ULH is comparatively easily obtainable in a chiral doped bent-core system. Once ULH is formed, the helicoidal axis (optic axis) lies along the rubbing direction. The electric field applied perpendicular to the substrate results in an in-plane deviation of the optic axis [17] and this follows and the sign of the direction of the field. For small electric fields $E$, the tilt angle $\phi$ is linearly related to the applied electric field by the expression

$$
\tan \phi=\frac{e^{\prime} p}{2 \pi K} E
$$

where $e^{\prime}$ and $K$ are the flexoelectric and effective elastic constants, respectively, defined as $e^{\prime}=\frac{\left|\left(e_{1}-e_{3}\right)\right|}{2}, K=\frac{K_{11}+K_{33}}{2}$; $e^{\prime}=e / 2, e$ is the effective flexoelectric coefficient; $p$ is the helicoidal pitch of the helix. Hence, one can calculate $\left|\left(e_{1}-e_{3}\right)\right|$ if the pitch, the splay, and bend elastic constants are known.

In order to obtain $\phi$ and hence $\left|\left(e_{1}-e_{3}\right)\right|$ from the ULH structure, C4, C6, and C7 are doped with a left-handed chiral 
dopant (S 811, E. Merck, Korea). The concentration range of the dopant was varied from $\sim 1.0 \%$ to $1.5 \%$ by weight, whereas the dopant has an extremely large helical twisting power of $58 \mu \mathrm{m}^{-1}$. These concentrations were chosen so as not to alter the properties of the compounds by the addition of the dopant, but it was also found that with higher weight percentage of the chiral additive $(\geqslant 2.5 \%)$, the optic axis especially in C6 did not respond to the electric field. The optical pitch of the helix was determined in a planar cell by the measurement of the selective reflection using a UV/Vis/IR Perkin-Elmer 900 spectrometer and using the formula $p=\frac{\lambda_{0}}{\langle n\rangle} ; \lambda_{0}$ is the wavelength of the selective reflection and $\langle n\rangle$ is the mean refractive index of the LC material, defined as $\left(n_{e}+n_{o}\right) / 2 ; n_{o}$ and $n_{e}$ are the ordinary and extraordinary refractive indices. The pitch length of the mixtures are determined and found to be $\sim 498,917$, and $930 \mathrm{~nm}$ for $\mathrm{C} 4, \mathrm{C} 6$, and $\mathrm{C} 7$, respectively. These were also found to be almost independent of temperature in the window of the experiment. The ULH texture is obtained by cooling a planar cell at a slow rate of $0.1{ }^{\circ} \mathrm{C} / \mathrm{min}$ from the isotropic into the cholesteric phase (just below the transition temperature) under the application of a moderate alternating electric field of $\sim 1 \mathrm{~V} / \mu \mathrm{m}$ at a frequency of $100 \mathrm{~Hz}$. The texture of the ULH structure is observed through a polarizing optical microscope (Olympus BX51) and is shown in Fig. 3 for a reduced temperature of $T-T_{\mathrm{NI}}=-4{ }^{\circ} \mathrm{C}$.

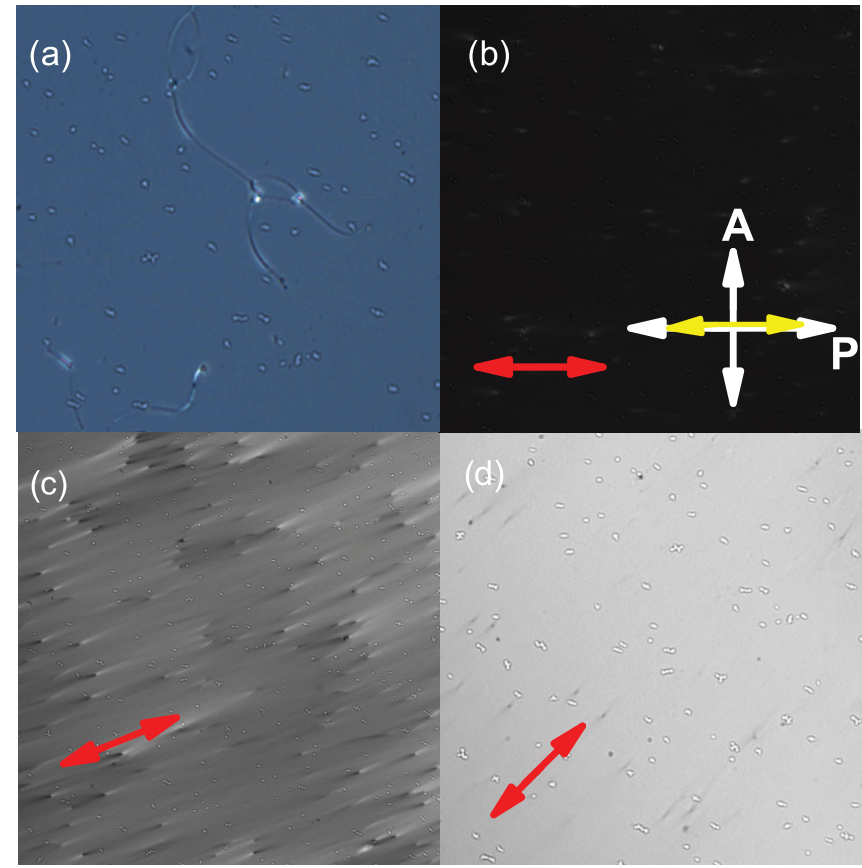

FIG. 3. (Color online) (a) Cholesteric texture obtained in a planar cell under crossed analyzer-polarizer (A and $\mathrm{P}$ ) with a chiral dopant added to $\mathrm{C} 4$ on cooling from the isotropic phase without field applied at a temperature $T-T_{\mathrm{NI}}=-4{ }^{\circ} \mathrm{C}$. On cooling the cell from the isotropic phase under the application of a moderate field, the ULH textures are obtained; the optic axis of the ULH lies parallel to the substrate in the direction of rubbing [shown by the yellow arrow in panel (b)]. ULH textures are shown in panels (b), (c), and (d) for different angles of rotation of the cell enclosed in the hot stage; the optic axis [depicted by the red arrows in panels (b), (c), and (d)] is aligned at angles of $0^{\circ}, 20^{\circ}$, and $45^{\circ}$ with respect to the polarizer $\mathrm{P}$.
The temperature of the sample in the hot stage is regulated using a temperature controller (Eurotherm). An electric field (square wave of frequency $20 \mathrm{~Hz}$ ) is then applied to the device; this causes an in-plane rotation of the optic axis due to the flexoelectric coupling. The response of the device to the field as sensed by a photodiode is recorded by a digitizing oscilloscope (Tektronics TDS 2014) for various positions of the sample with respect to crossed polarizers, with an angular step of $10^{\circ}$. The maximum and the minimum amplitudes of the signal, $U_{\max }$ $(+E)$ and $U_{\min }(-E)$ at a particular instant in time are then plotted as a function of the rotation angle, $\theta$. This generates a periodic signal in time, fitted to a sine-wave function for minimizing errors in the data collection. The phase difference between the two waves is equal to $2 \phi$ (demonstrated later in Fig. 10), from which the tilt angle of the optic axis is easily determined. Measurements are carried out for fields much below those required for helical unwinding and so as not to damage the cells when subjected to higher fields.

\section{RESULTS AND DISCUSSIONS}

\section{A. Dielectric measurements}

The dielectric data obtained for $\mathrm{C} 6$ at a frequency of $1 \mathrm{kHz}$ as a function of reduced temperature $T-T_{\mathrm{NI}}$ are plotted in Fig. 4. For higher temperatures, $\Delta \varepsilon \leqslant+0.3$, but as the temperature is reduced further, $\Delta \varepsilon$ turns out to be negative below a crossover temperature of $C_{\mathrm{T}}$. The dielectric anisotropy is low enough but is ideal for minimizing errors in the determination of the flexoelectric coefficient.

The crossover temperature where the dielectric anisotropy changes sign is easily determined for C6 from the dielectric data. The nematic phase for C6 is found to extend over a wide range of temperatures. However, $\mathrm{C} 4$ when kept at lower temperatures (but higher than the nematic-crystalline transition temperature) tends to crystallize with the passage of time. Figure 5 shows how the perpendicular component of

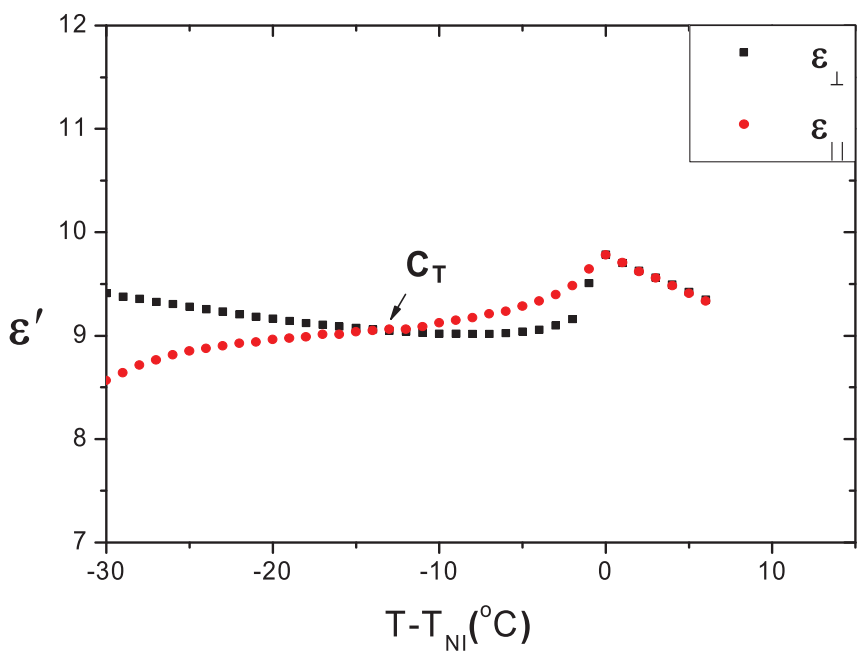

FIG. 4. (Color online) Dielectric anisotropy of C6 as a function of the reduced temperature $T-T_{\mathrm{NI}}\left({ }^{\circ} \mathrm{C}\right), T_{\mathrm{NI}}$ is the N-I transition temperature; permittivity is measured at a frequency of $1 \mathrm{kHz} . C_{\mathrm{T}}$ is the temperature at which the two curves cross over each other. Red dots are $\varepsilon_{\|}$and black squares are $\varepsilon_{\perp}$. 


\section{C4 BAN Planar cell}

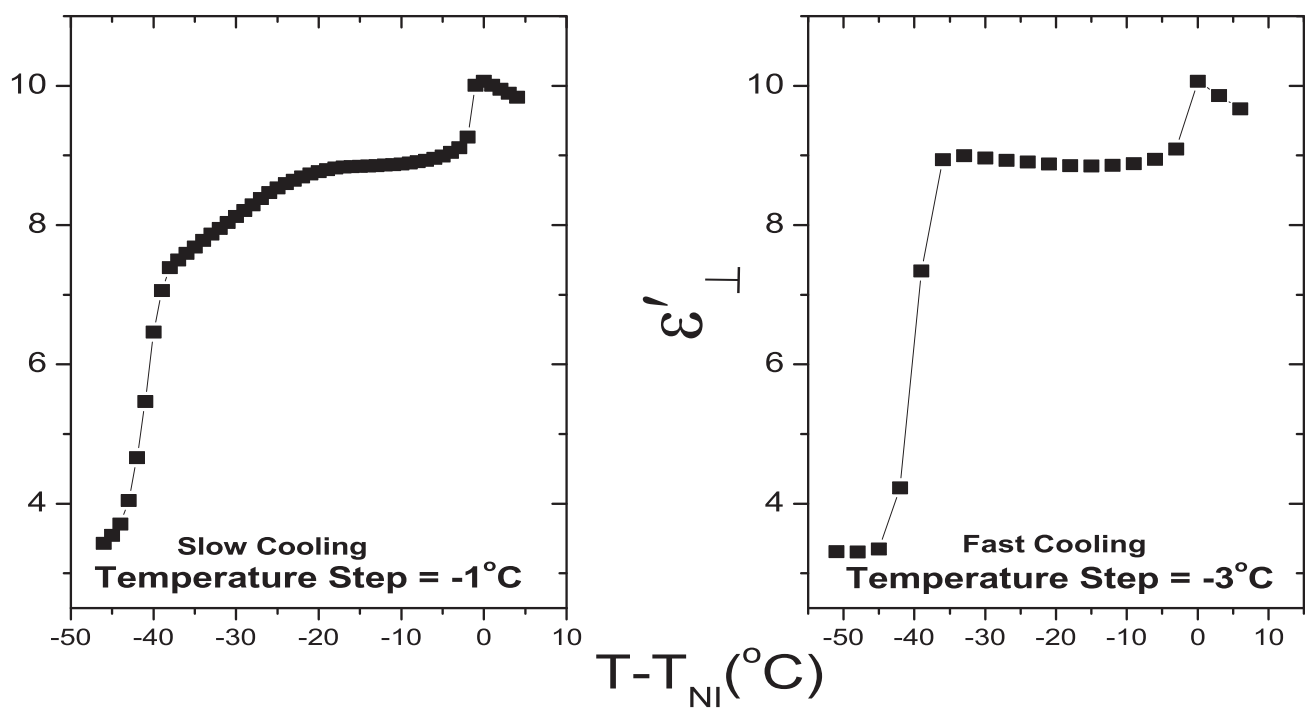

FIG. 5. Dielectric permittivity of $\mathrm{C} 4$ shows a drop in the value of the perpendicular component of $\varepsilon^{\prime}$ at a frequency of $1 \mathrm{kHz}$ due to crystallization of the material at lower temperatures. Data in (a) on the left are recorded with a temperature step of $-1{ }^{\circ} \mathrm{C}$ and in (b) on the right with a temperature step of $-3{ }^{\circ} \mathrm{C}$ (faster cooling). Each temperature point takes 6 to 8 min to scan. In Ref. [7], the cooling rate was faster, $10 \mathrm{~K} / \mathrm{min}$, and the boundary conditions for DSC were different and hence crystallization was not observed there.

permittivity, determined from a planar cell, decreases at lower temperatures due to the slow crystallization of the material much before the onset of the crystalline phase. Hence the optical measurements for $\mathrm{C} 4$, carried out in planar cells, could only be made at temperatures where it does not crystallize with time. The parallel component of the permittivity $\varepsilon_{\|}$in C4 (obtained from a homeotropic cell) is observed to increase just below the isotropic-nematic transition temperature. As temperature is further lowered $\varepsilon_{\|}$is seen to decrease steadily as the crossover temperature is approached.

A change in the sign of the dielectric anisotropy observed using dielectric spectroscopy is also confirmed by the optical transmittance technique, known for its higher reliability, especially when ions are present in a liquid crystalline medium. Here the planar cell is cooled down with a constant field [9.5 $\mathrm{V}(\sim 2 \mathrm{~V} / \mu \mathrm{m}), 1 \mathrm{kHz}$ sine wave] applied across it from the isotropic phase, and change in the transmission is recorded as a function of temperature [22]. The setup consisted of a Leitz polarizing microscope, photodiode (Hamamatsu C6386), and a data acquisition board (NI-USB-6216) interfaced with a computer. When an electric field much greater than the threshold voltage $\left(V_{t h}\right)$ is applied to the cell in a temperature region where $\Delta \varepsilon>0$, Freederickz transition occurs, leading to a drastic change in the intensity observed through the crossed polarizers. In the temperature ranges where $\Delta \varepsilon<0$, no Freederickz transition was observed and the alignment remained planar. Figure 6 shows the transmittance as a function of the reduced temperature for $\mathrm{C} 4$ and C6. The dashed line indicates a sharp transmittance change closer to the temperature where the dielectric anisotropy changes its sign. Although this technique may not give the exact crossover temperature and the value of $\Delta \varepsilon$, it is effective in determining its sign reversal.

Detailed dielectric studies on C7 BAN, also showing sign reversal of $\Delta \varepsilon$, were reported by Jang et al. [22]. Further studies by Jang et al. [23] of this series of cyanoresorcinols revealed that the sign reversal of $\Delta \varepsilon$ as a function of frequency was related to the relaxation of the longitudinal component of the dipole moment occurring at much lower frequencies compared to the transverse component. The temperature dependence of permittivity was found to be related to a change in the strong anisotropic correlations among the molecules taking place with temperature. They ruled out the possibility of conformational change (used to describe the sign reversal phenomena in calamitic molecules) affecting the value of $\Delta \varepsilon$ by a change in the bent angle and the dipole moments [23].

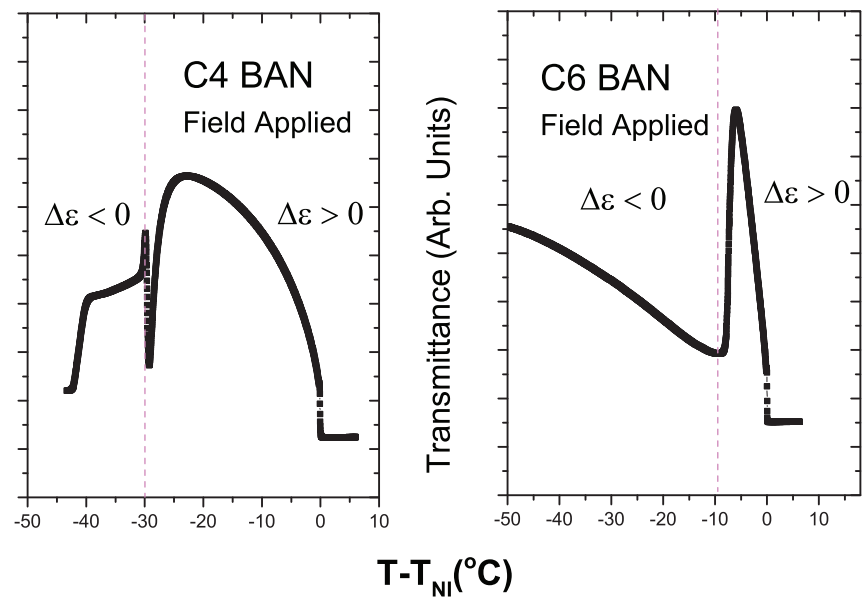

FIG. 6. (Color online) The variation of transmittance through a planar cell under crossed polarizers as a function of the reduced temperature for C4 BAN (left) and C6 BAN (right) at a field of $9.5 \mathrm{~V}$; sinusoidal signal frequency of $1 \mathrm{kHz}$ is applied across the cell. There is a changeover in the intensity close to the Freederickz transition. Waiting time between each data point was kept to $\sim 2 \mathrm{~s}$. 
$\Delta \varepsilon$ values for bent-core systems in the nematic phase are unusually low for the reason that dipole moments along the bow and the long axes are comparable to each other. It has also been experimentally demonstrated in the literature that $\Delta \varepsilon$ in mixtures of rodlike and $\mathrm{BCs}$ decreases as the concentration of the $\mathrm{BC}$ molecules is increased [42].

Due to a low positive value of $\Delta \varepsilon$ for C6 (Fig. 4) observed over a narrow range of temperatures [Fig. 6(b)] below the I-N (cholesteric) phase transition, flexoelastic studies are thus restricted to only such a narrow range of temperatures only. A supercooling effect observed in $\mathrm{C} 4$ (Fig. 5) also restricts our studies to a narrow range of temperatures in this as well.

\section{B. Results on the elastic constants}

The splay and bend elastic constants in the nematic phase are determined from the voltage dependent retardation data using the method described earlier in Sec. III B. An example of the theoretical fit to the retardation curve for a reduced temperature, $T-T_{\mathrm{NI}}=-6^{\circ} \mathrm{C}$ for C6 BAN is shown in Fig. 7, where $T_{\mathrm{NI}}$ is the nematic-isotropic transition temperature. The temperature variation of the splay and bend elastic constants found using the procedure described here are plotted in Fig. 8 for the three compounds. $K_{11}$ is seen to increase with a reduction in temperature and is almost linear for $\mathrm{C} 6$ where the value increases from $\sim 3.97 \mathrm{pN}$ at $2{ }^{\circ} \mathrm{C}$ below the I-N transition to $8.81 \mathrm{pN}$ at the lowest reduced temperature, whereas changes are less subtle for $\mathrm{C} 4$ and $\mathrm{C} 7$, where it increases from 3.44 to $5.05 \mathrm{pN}$ and $\sim 3.89$ to $9.98 \mathrm{pN}$, respectively, as the temperature is lowered. The maximum splay elastic constant $K_{11}$ for C6 is found to be almost 2.2 times its lowest value, while for C4 the increase in $K_{11}$ is $\sim 1.5$ times and is almost $\sim 2.56$ for $\mathrm{C} 7$ for the lowest temperature measured. On the other hand, $K_{33}$ is less than $\mathrm{K}_{11}$ for all the compounds studied. The results, in general, agree with other BCN [24,33] systems. The ratio of $K_{33} / K_{11}$ for C6 is seen to decrease from $\sim 0.8$ at $T-T_{\mathrm{NI}}=-2{ }^{\circ} \mathrm{C}$ to 0.45 at $T-T_{\mathrm{NI}}=-7{ }^{\circ} \mathrm{C}$, mainly due to an increase in $K_{11}$ with a reduction in temperature. For $\mathrm{C} 4$ the

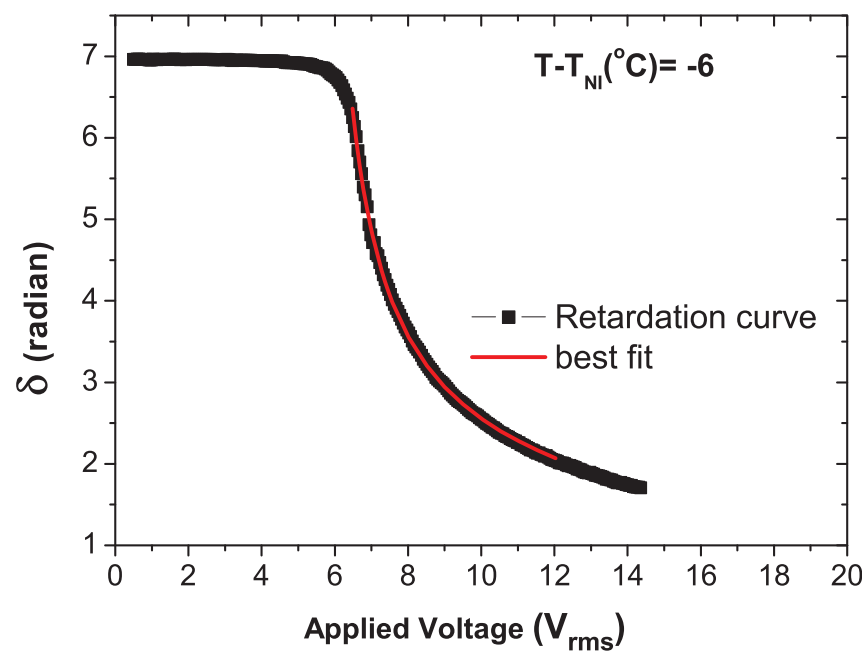

FIG. 7. (Color online) Retardation as a function of applied voltage for $\mathrm{C} 6 \mathrm{BAN}$ for a reduced temperature of $-6^{\circ} \mathrm{C}$. The continuous red line is the theoretical fit to Eqs. (4) and (5) that are used to determine $K_{33}$.
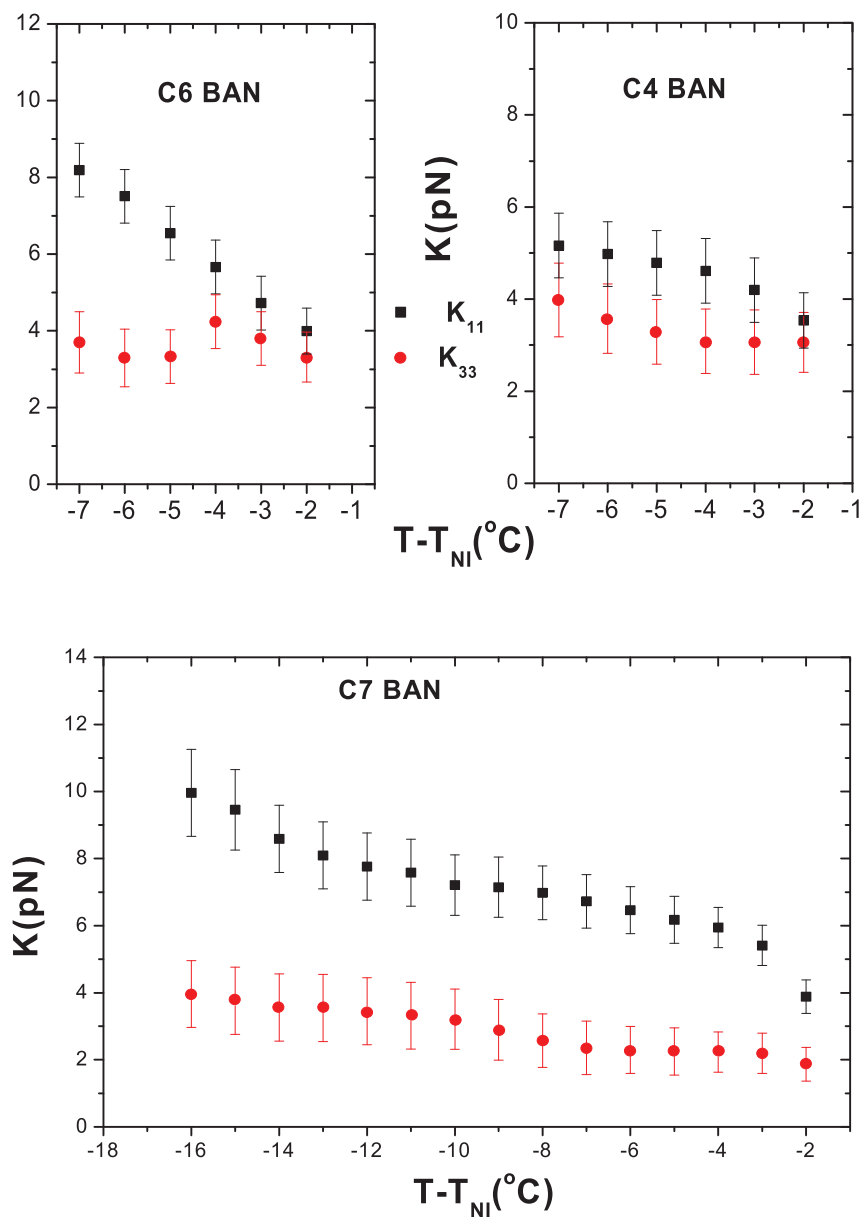

FIG. 8. (Color online) Splay $K_{11}$ and bend $K_{33}$ elastic constants as a function of the reduced temperature; C6 BAN (top left), C4 BAN (top right), and C7 BAN (bottom). The symbols with the bars denote the maximum possible errors present in the calculation due to the absence of a sharp threshold voltage $V_{t h}$ and the errors involved in normalization of temperature between different setups used in finding different parameters for calculating $K_{11}$ and $K_{33}$ using the iterative procedure given in Ref. [35].

ratio only decreases from $\sim 0.85$ to 0.78 as the temperature is lowered, while for $\mathrm{C} 7$ the ratio is also seen to vary slightly from $\sim 0.46$ to 0.39 in the temperature range measured. The trend nevertheless is different than those found for calamitic nematics [27-29]. The bend elastic constant is found to be nearly independent of temperature for $\mathrm{C} 6$, whereas for $\mathrm{C} 4$ and $\mathrm{C} 7$ it increases marginally with a reduction in temperature. The elastic constants for C6, determined previously by Salter et al. [40], are slightly lower than ours. They obtained results from the dielectric permittivity data, whereas we fit the corresponding optical retardation data to the field. The optical technique is found to be better than the dielectric one especially when ions are present in the medium. It is well known that ions in LCs do not affect the optical transmittance through a cell to the same extent as these do the dielectric measurements.

The effective elastic constant $K$ is plotted as a function of the reduced temperature in Fig. 9. For the materials studied, $K$ is seen to increase with a reduction in temperature and values for $\mathrm{C} 6$ are somewhat greater than for $\mathrm{C} 4$ and $\mathrm{C} 7$, in a common temperature range for which $K$ was determined, i.e., 


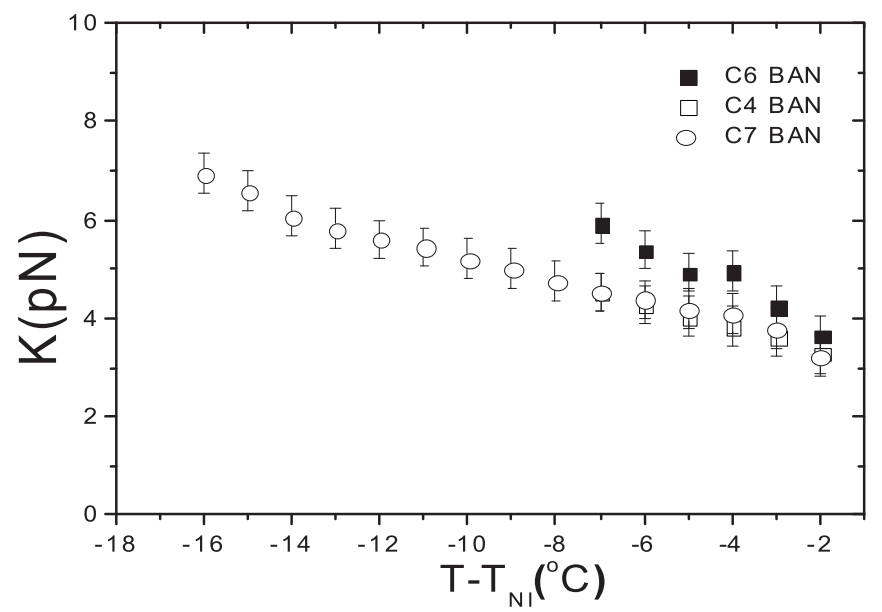

FIG. 9. Plot of the effective elastic constant $K$ as a function of the reduced temperature. Solid squares ( $\boldsymbol{\square})$ represent the data for C6, open squares ( $\square$ ) for $\mathrm{C} 4$, and open circles (o) for C7. Results for C4, $\mathrm{C} 6$, and $\mathrm{C} 7$ are not very different from each other.

between $T-T_{\mathrm{NI}}=-2{ }^{\circ} \mathrm{C}$ and $-7^{\circ} \mathrm{C}$. The effective flexoelastic coefficient $e / K$ is an important characteristic parameter for a device based on flexoelectricity, since $\tan \phi \propto \frac{e}{K}$ from Eq. (6); hence, a lower $K$ and a higher $e$ imply a larger value of the flexo-optic tilt angle, $\phi$. This increases the potential of the flexoelectric based devices using BCNs.

For an oxadiazole bent-core compound, Kaur et al. [43] found $K_{33}<K_{11}$. They explained their observations by the molecular field theory and atomistic modeling, suggesting that $K_{11}$ is almost independent of the molecular conformation, whereas $K_{33}$ is sensitive to changes in the molecular curvature, similar to those calculated for odd- dimeric mesogens $[35,44]$. The elastic constants measured in some odd-dimeric compounds show similar trends as in BC systems, where $K_{33}<K_{11}$ with $K_{33}$ decreasing steadily as temperature is reduced [35]. Our results for the three bent-core systems show that even in the absence of significant cybotactic clusters in $\mathrm{C} 4, K_{33}$ is lower than $K_{11}$. We can thus safely state that in BCNs $K_{33}<K_{11}$ is due to the bent shape of the molecules rather than arising from the presence of clusters. Our results likewise can be explained by the theory and modeling proposed by Kaur et al. [43] and Cestari et al. [44]. The bent shape favors the bend distortions of the director as opposed to splay. The increase in $K_{11}$ for $\mathrm{C} 6 \geqslant \mathrm{C} 7>\mathrm{C} 4$ seems to arise from the longer molecular chain lengths in $\mathrm{C} 6$ and $\mathrm{C} 7$ than from clusters being present from the nematic phase of $\mathrm{C} 6$ and $\mathrm{C} 7$ but much smaller or absent in $\mathrm{C} 4$.

\section{Flexoelectric coefficients}

An example of the measurement of the tilt angle of the optic axis in ULH of $\mathrm{C} 4$ at a temperature of $T-T_{\mathrm{NI}}=$ $-2{ }^{\circ} \mathrm{C}$ and applied field of $0.6 \mathrm{~V} / \mu \mathrm{m}$ is shown in Fig. 10 . The tilt angle of the optic axis of ULH as a function of the applied electric field is plotted in Fig. 11 and is found to vary linearly with the field but surprisingly is found to be almost independent of temperature, as seen in Fig. 11. This means that both $e$ and $K$ depend on temperature similarly and both follow $S^{2}$ dependence, where $S$ is the orientational order parameter. The conformational freedom of molecules is thus restricted and this agrees with the conclusions derived from

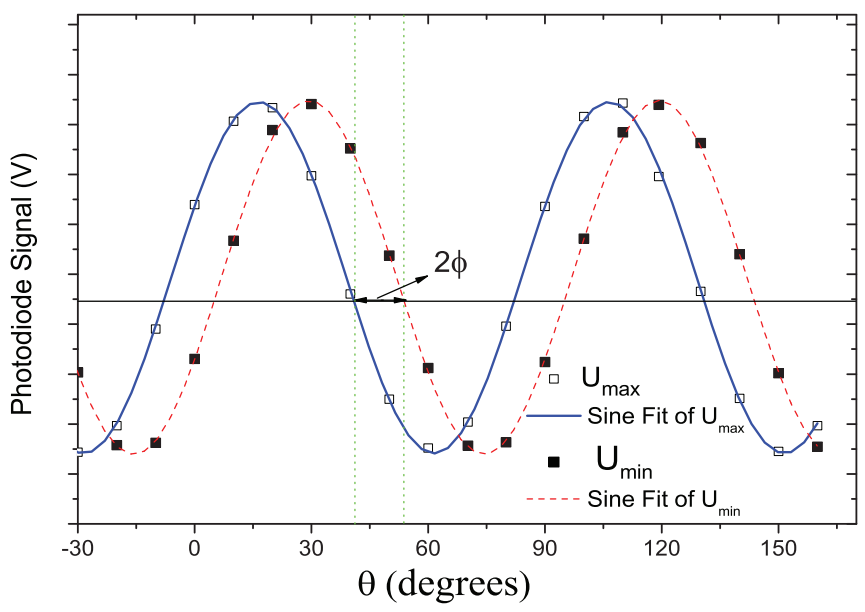

FIG. 10. (Color online) The photodiode signal at a fixed point (in our case time) as a function of sample rotation angle, $\theta$ for $\mathrm{C} 4$ at $T-T_{\mathrm{NI}}=-2{ }^{\circ} \mathrm{C}$ for a field of $0.6 \times 10^{6} \mathrm{~V} / \mu \mathrm{m}$. The open squares ( $\square$ ) represent the maximum amplitude $U_{\max }$ of the ULH signal and the solid squares (ם) represent the minimum amplitude, $U_{\min }$. The blue and red lines are the best fits of curves for $U_{\max }$ and $U_{\min }$, respectively. The difference between the two signals as marked in the figure corresponds to $2 \phi$ in degrees.

dielectric studies reported by Jang et al. [23]. The magnitude of the effective flexoelectric coefficient $\left|\left(e_{1}-e_{3}\right)\right|$ is obtained using Eq. (6), by knowing the experimental values of the pitch and angle $\phi$ for an applied field $E$. Data are shown in Fig. 12. For $\mathrm{C} 4, \mathrm{C} 6$, and $\mathrm{C} 7$, it is found that the magnitude of $\left|\left(e_{1}-e_{3}\right)\right|$ increases with a reduction in temperature. For $\mathrm{C} 4,\left|\left(e_{1}-e_{3}\right)\right|$ increases from $14 \mathrm{pC} / \mathrm{m}$ for $T-T_{\mathrm{NI}}=-2{ }^{\circ} \mathrm{C}$ to $\sim 23 \mathrm{pC} / \mathrm{m}$ at the lowest reduced temperature, while for $\mathrm{C} 6$ it increases from 11 to $19.5 \mathrm{pC} / \mathrm{m}$ and for $\mathrm{C} 7$ it increases from $\sim 10.89$ at $T-T_{\mathrm{NI}}=-2{ }^{\circ} \mathrm{C}$ to $24.4 \mathrm{pC} / \mathrm{m}$ at $T-T_{\mathrm{NI}}=-16^{\circ} \mathrm{C}$.

The effective flexoelectric coefficient for C4, C6, and C7 is greater than reported for the conventional rodlike molecules that have similar magnitudes of the dipole moments $[39,45]$, but its order of magnitude is comparable to the other BCNs $[24,40,43]$. However, it is much lower than that found by Harden et al. [9] for another BCN LC. While Kumar et al. found the value of $\left|e_{3}\right|$ comparable to those of the conventional nematics, nevertheless they proposed a model of nonpolar clusters that would yield quadrapolar flexoelectricity [24] to explain the giant flexoelectricity in bent-core systems observed by Harden et al. [9]. They state that a giant value in the flexoelectric coefficient if confirmed can, in principle, be attributed to the nonpolar clusters formed in the nematic phase. The flexoelectric coefficient should scale as the second power of the number of clustered molecules.

$\left|\left(e_{1}-e_{3}\right)\right|$ determined by Salter et al. [40] for C6 though slightly lower than ours is of comparable magnitude and this difference arises most likely from using a different value of the effective elastic constant in Eq. (6) as a function of temperature. Due to the presence of SmC-like clusters in the entire nematic phase of $\mathrm{C} 6$ and $\mathrm{C} 7$, we should expect larger values of $\left|\left(e_{1}-e_{3}\right)\right|$ in comparison to $\mathrm{C} 4$ and furthermore these values should increase significantly with a reduction in temperature if the model of Kumar et al. were to hold good [24], 

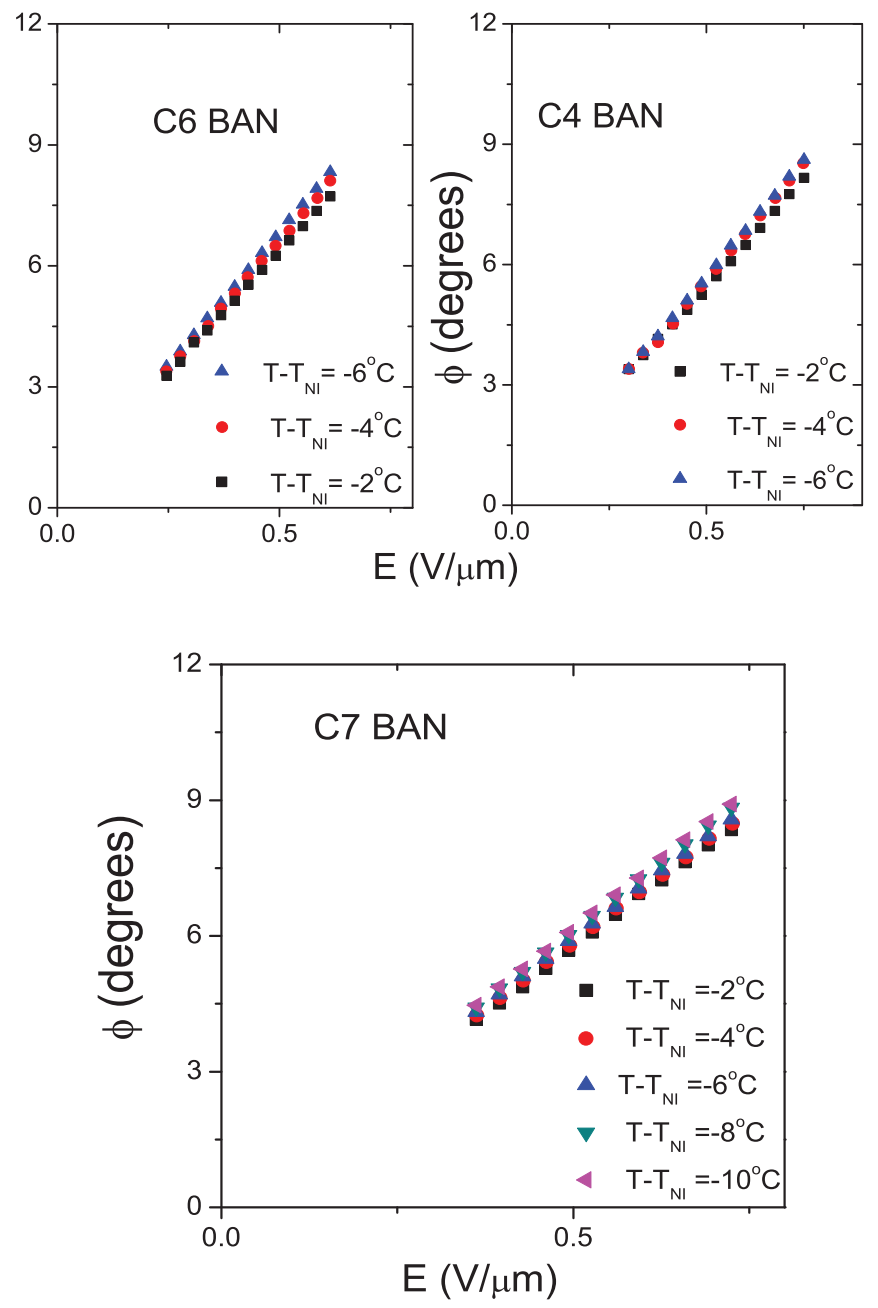

FIG. 11. (Color online) The magnitude of the tilt of the optic axis in the ULH structure of C6 BAN (top left), C4 BAN (top right), and C7 BAN (bottom) as a function of the applied electric field $E$, for selected reduced temperatures given in the Figure. An explanation for the different symbols is also given.

since the size of the cluster increases significantly. However, our experimental results disagree with their conjecture. We conclude that the flexoelectric coefficient in BCN systems, though larger by a factor of up to 4 than calamatics [39], is not giant and an increase by a factor of 1000 is being ruled out here. It may be mentioned that Kumar et al. [24] obtained even lower values of the flexoelectric coefficients for BCNs than determined by us in spite of their having proposed the model of the giant flexoelectricity. They determined both $e_{1}$ and $e_{3}$ for the two different bent-core systems with both positive and negative dielectric anisotropies using the method initially suggested by Helfrich [12]. Results on the bent-core systems C4, C6, and C7 nevertheless show that the clusters, by and large, do not affect the elastic constants or the effective flexoelectric coefficients to any significant extent.

The effective flexoelectric coefficient in this work is determined by applying a square-wave signal of frequency of $20 \mathrm{~Hz}$ across the cell, the axis of the ULH is displaced in the plane of the cell and the displacement follows the alterations of the signal. This frequency of the applied field is somewhat
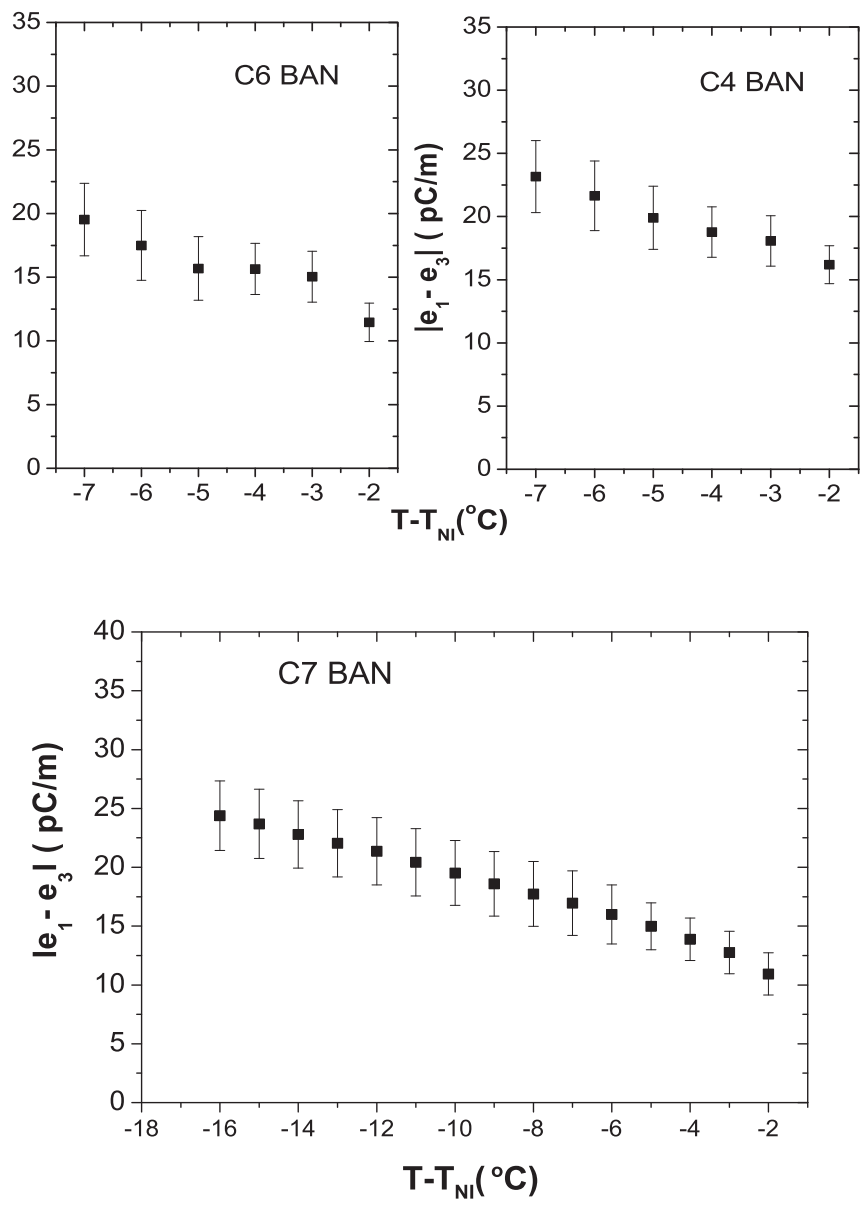

FIG. 12. The magnitude of the difference of the flexoelectric coefficients $\left|\left(e_{1}-e_{3}\right)\right|$ plotted as a function of the reduced temperature. The top left panel shows results for C6 BAN, the top right panel for C4 BAN, and the bottom panel for C7 BAN. The reason for the error bars shown on the data points is given in the text.

higher than that used by Harden et al. [9], who used frequency varying from 1 to $10 \mathrm{~Hz}$. In any case, the signal of $20 \mathrm{~Hz}$ should be sufficient to switch the clusters. For most members of this homologous series (C4, C7, and C9), Jang et al. [23] have reported the dielectric permittivity spectra in the frequency range $1 \mathrm{kHz}$ to $1 \mathrm{MHz}$, where they observed the various dielectric modes. It was, however, not possible to extend this study to frequencies lower than $1 \mathrm{kHz}$ as the ions significantly affected the results of dielectric permittivity. The presence of ionic impurity is a continuing problem in BCNs and can be solved presumably through a better purification/synthetic procedure.

As suggested by Salter $e t$ al. [40], one should also measure $\left|e_{1}+e_{3}\right|$ in addition to having measured $|e|=\left|e_{1}-e_{3}\right|$; then one may calculate $\left|e_{3}\right|$ and it may turn out to be much greater than $|e|$. However, the outcome of this experiment is not promising as Takezoe et al. [46] have already found $\left|e_{3}\right|$ for the same compound as studied by Harden et al. [9]. Takezoe et al. [46] found the value $15.8 \mathrm{pC} / \mathrm{m}$ using an experimental method as well as simulated using Eq. (2). The explanation of the simulated parameters of the compound is given in [46]. The only difference in the approach therefore lies in using a direct flexoelectric effect by Harden et al. [9] rather than using 
a converse flexoelectric effect by other studies. Harden et al. do not discuss the limitations of their method as to whether or not the ionic impurities or electroconvection and or rupturing of the director field by relative movements of two substrates or mechano-convection affect their results. The results reported in our paper imply that the effect of the quadrupolar order on the measurement of the effective flexoelectric coefficient $\left|\left(e_{1}-e_{3}\right)\right|$ is not being cancelled out.

\section{CONCLUSIONS}

The elastic constant $K_{11}$ is larger for C6 and C7 than for $\mathrm{C} 4$ by a factor of $\sim 1.6$ depending on the temperature. This presumably is due to longer chain lengths for C6 and C7 rather than arising from the presence of clusters in $\mathrm{C} 6$ and $\mathrm{C} 7$. At a temperature of $7{ }^{\circ} \mathrm{C}$ below the I-N transition temperature, $K_{11}$ for $\mathrm{C} 7$ is $\sim 7 \mathrm{pN}$, for $\mathrm{C} 6$ is $\sim 8 \mathrm{pN}$, and for $\mathrm{C} 4$ is $\sim 5 \mathrm{pN}$. $K_{33}$ for three BCNs (C4, C6, and C7) similarly are found to be low and almost temperature independent. For C4 and C6, $K_{33}$ are approximately the same but somewhat greater than for $\mathrm{C} 7$. Such low values of $K_{33}$ seem to arise from the bent shape of the molecules rather than having been caused by the presence or absence of clusters. If the clusters were to have an effect, these could only tend to lower $K_{33}$.

The experimental results on the flexoelectric coefficient $\left|\left(e_{1}-e_{3}\right)\right|$ for the three symmetric bent-core LCs C4, C6, and $\mathrm{C} 7$ of the same homologous series of cyanoresorcinol bisbenzoates have been obtained. A ULH configuration is obtained by adding a small percentage of left-handed chiral dopant (S811 E Merck, Korea) with a large twisting power to these bent-core systems and cooling slowly under a moderate electric field to cholesteric phase just below the isotropic phase. The effective flexoelectric coefficient has a negative sign and follows the convention given by Castles et al. [39] and its magnitude $|e|$ is observed to be greater by a factor up to 4 than for calamatics [39], determined by applying a square wave signal of frequency $20 \mathrm{~Hz}$, but it is much lower than anticipated for bent-core systems from the results of Harden et al. [9]. X-ray studies reveal that $\mathrm{SmC}$ type clusters are present in the entire nematic range for $\mathrm{C} 6$ and $\mathrm{C} 7$ but are measurably absent in $\mathrm{C} 4$. When we compare the effective flexoelectric coefficients of the three compounds, $\left|\left(e_{1}-e_{3}\right)\right|$ is found to be higher for $\mathrm{C} 4$ by $20 \%$ to $25 \%$ at a given reduced temperature than for $\mathrm{C} 6$ and $\mathrm{C} 7$. If clusters are thought to be responsible for increasing the effective flexoelectric coefficient, then our results contradict these. The molecular shape, the chain lengths of the bent-core molecule, and its transverse dipole moment when considered together can account for these results.

\section{ACKNOWLEDGMENTS}

JKV work in Sungkyunkwan, Suwon, Korea (2012-13) was supported by World Class University program through the National Research Foundation of Korea funded by the Ministry of Education, Science and Technology (R32-2008-000-10204-0). We thank Jang-Kun Song for a gift of the chiral dopant S811 and for valuable discussions. One of the authors (RB) thanks IRCSET for the award of a research studentship. Collaboration between Dublin and Halle was funded by the EU Bind Project 2008-12 (Grant No. 216025) for which we thank Demetri J. Photinos for coordination and useful discussions. We also thank Steve J. Elston for recasting Eq. (3).
[1] D. Vorländer, Ber. Dtsch. Chem. Ges. 62, 2831 (1929); D. Vorländer and A. Apel, ibid. 65, 1101 (1932).

[2] T. Niori, T. Sekine, J. Watanabe, T. Furukawa, and H. Takezoe, J. Mater. Chem. 6, 1231 (1996).

[3] D. R. Link, G. Natale, R. Shao, J. E. Maclennan, N. A. Clark, E. Korblova, and D. M. Walba, Science 278, 1924 (1997).

[4] H. Takezoe and Y. Takanishi, Jpn. J. Appl. Phys. 45, 597 (2006); R. A. Reddy and C. Tschierske, J. Mater. Chem. 16, 907 (2006).

[5] C. Keith, M. Prehm, Y. P. Panarin, J. K. Vij, and C. Tschierske, Chem. Commun. 46, 372 (2010).

[6] D. Shen, S. Diele, G. Pelzl, I. Worth, and C. Tschierske, J. Mater. Chem. 9, 661 (1999); J. Matraszek, J. Mieczkowski, J. Szydlowska, and E. Gorecka, Liq. Cryst. 27, 429 (2000); T. J. Dingemans and E. T. Samulski, ibid. 27, 131 (2000); I. Wirth, S. Diele, A. Eremin, G. Pelzl, S. Grande, L. L. Kovelenko, N. Pancenko, and W. Weissflog, J. Mater. Chem. 11, 1642 (2001).

[7] C. Keith, A. Lehmann, U. Baumeister, M. Prehm, and C. Tschierske, Soft Matter 6, 1704 (2010).

[8] A. Jákli, M. Chambers, J. Harden, M. Madhabi, R. Teeling, J. Kim, Q. Li, G. G. Nair, N. Éber, K. Fodor-Csorba, J. T. Gleeson, and S. Sprunt, Proc. SPIE 6911, 691105 (2008).

[9] J. Harden, B. Mbanga, N. Éber, K. Fodor-Csorba, S. Sprunt, J. T. Gleeson, and A. Jákli, Phys. Rev. Lett. 97, 157802 (2006).

[10] R. B. Meyer, Phys. Rev. Lett. 22, 918 (1969).

[11] F. Castles, S. M. Morris, and H. J. Coles, AIP Adv. 1, 032120 (2011).
[12] W. Helfrich, Phys. Lett. A 35, 393 (1971); Z. Naturforsch. 26a, 833 (1971).

[13] P. Palffy-Muhoray, AIP Adv. 3, 019101 (2013); F. Castles, S. M. Morris, and H. J. Coles, ibid. 3, 019102 (2013).

[14] D. Dunmur, A. Fukuda, and G. R. Luckhurst, Physical Properties of Liquid Crystals: Nematics (Institution of Engineering and Technology, London, UK, 2001).

[15] A. Derzhanski, A. G. Petrov, and M. D. Mitov, J. Phys. (France) 39, 273 (1978).

[16] L. A. Parry-Jones, R. B. Meyer, and S. J. Elston, J. Appl. Phys. 106, 014510 (2009). This equation initially taken from Ref. 15 is corrected here.

[17] J. S. Patel and R. B. Meyer, Phys. Rev. Lett. 58, 1538 (1987).

[18] P. J. Bos and K. R. Koehler/beran, Mol. Cryst. Liq. Cryst. 113, 329 (1984); S. A. Jewell and J. R. Sambles, J. Appl. Phys. 92, 19 (2002).

[19] B. R. Acharya, A. Primark, and S. Kumar, Phys. Rev. Lett. 92, 145506 (2004); L. A. Madsen, T. J. Dingemans, M. Nakata, and E. T. Samulski, ibid. 92, 145505 (2004).

[20] Y. Jang, V. P. Panov, A. Kocot, J. K. Vij, A. Lehmann, and C. Tschierske, Appl. Phys. Lett. 95, 183304 (2009).

[21] M. Nagaraj, Y. P. Panarin, U. Manna, J. K. Vij, C. Keith, and C. Tschierske, Appl. Phys. Lett. 96, 011106 (2010); M. Nagaraj, K. Merkel, J. K. Vij, and A. Kocot, Euro. Phys. Lett. 91, 66002 (2010). 
[22] Y. Jang, V. P. Panov, C. Keith, C. Tschierske, and J. K. Vij, Appl. Phys. Lett. 97, 152903 (2010).

[23] Y. Jang, V. P. Panov, A. Kocot, A. Lehmann, C. Tschierske, and J. K. Vij, Phys. Rev. E 84, 060701(R) (2011).

[24] P. Kumar, Y. G. Marinov, H. P. Hinov, U. S. Hiremath, C. V. Yelamaggad, K. S. Krishnamurthy, and A. G. Petrov, J. Phys. Chem. B 113, 9168 (2009).

[25] M. Majumdar, P. Salamon, A. Jákli, J. T. Gleeson, and S. Sprunt, Phys. Rev. E 83, 031701 (2011).

[26] Y. Jang, R. Balachandran, C. Keith, A. Lehmann, C. Tschierske, and J. K. Vij, Soft Matter 8, 10479 (2012); Y. Jang, Ph.D. thesis, University of Dublin Trinity College, Dublin, Ireland, 2012.

[27] G.-P. Chen, H. Takezoe, and A. Fukuda, Liq. Cryst. 5, 341 (1989).

[28] W. H. de Jeu and W. A. P. Claassen, J. Chem. Phys. 67, 8 (1977); A. Scharkowski, H. Schmiedel, R. Stannarius, and E. Weisshunn, Mol. Cryst. Liq. Cryst. 191, 1 (1990).

[29] M. Schadt, R. Buchecker, F. Leenhouts, A. Boller, A. Villiger, and M. Petrzilka, Mol. Cryst. Liq. Cryst. 139, 1 (1986).

[30] M. R. Dodge, C. Rosenblatt, R. G. Petschek, M. E. Neubert, and M. E. Walsh, Phys. Rev. E 62, 5056 (2000).

[31] M. R. Dodge, R. G. Petschek, C. Rosenblatt, M. E. Neubert, and M. E. Walsh, Phys. Rev. E 68, 031703 (2003).

[32] B. Kundu, R. Pratibha, and N.V. Madhusudana, Phys. Rev. Lett. 99, 247802 (2007).

[33] P. Sathyanarayana, M. Mathew, Q. Li, V. S. S. Sastry, B. Kundu, K. V. Le, H. Takezoe, and S. Dhara, Phys. Rev. E 81, 010702(R) (2010).
[34] P. Tadapatri, U. S. Hiremath, C. V. Yelamaggad, and K. S. Krishnamurthy, J. Phys. Chem. B 114, 1745 (2010).

[35] R. Balachandran, V. P. Panov, J. K. Vij, A. Kocot, M. G. Tamba, A. Kohlmeier, and G. H. Mehl, Liq. Cryst. 40, 681 (2013).

[36] H. J. Deuling, Mol. Cryst. Liq. Cryst. 19, 123 (1972).

[37] G. Barbara and L. R. Evangelista, An Elementary Course on the Continuum Theory of Nematic Liquid Crystals (World Scientific, Singapore, 2000); S. W. Morris, P. Palffy-Muhoray, and D. A. Balzarini, Mol. Cryst. Liq. Cryst. 139, 263 (1986).

[38] S.-D. Lee and J. S. Patel, Phys. Rev. A 42, 997 (1990).

[39] F. Castles, S. C. Green, D. J. Gardiner, S. M. Morris, and H. J. Coles, AIP Adv. 2, 022137 (2012).

[40] P. S. Salter, C. Tschierske, S. J. Elston, and E. P. Raynes, Phys. Rev. E 84, 031708 (2011).

[41] S. M. Morris, M. J. Clark, A. E. Blatch, and H. J. Coles, Phys. Rev. E 75, 041701 (2007).

[42] P. Sathyanarayana, V. R. S. Jampani, M. Skarabot, I. Musevič, K. V. Le, H. Takezoe, and S. Dhara, Phys. Rev. E 85, 011702 (2012).

[43] S. Kaur, J. Addis, C. Greco, A. Ferrarini, V. Görtz, J. W. Goodby, and H. F. Gleeson, Phys. Rev. E 86, 041703 (2012).

[44] M. Cestari, E. Frezza, A. Ferrarini, and G. R. Luckhurst, J. Mater. Chem. 21, 12303 (2011).

[45] P. S. Salter, C. Kischka, S. J. Elston, and E. P. Raynes, Liq. Cryst. 36, 1355 (2009).

[46] K. V. Le, F. Araoka, K. Fodor-Csorba, K. Ishikawa, and H. Takezoe, Liq. Cryst. 36, 1119 (2009). 